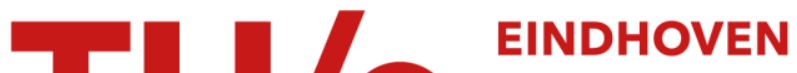 \\ UNIVERSITY OF \\ TECHNOLOGY
}

\section{Accurate determination of elastic parameters for multicomponent membranes}

\section{Citation for published version (APA):}

Semrau, S., Idema, T., Holtzer, L., Schmidt, T., \& Storm, C. (2008). Accurate determination of elastic parameters for multicomponent membranes. Physical Review Letters, 100(8), 088101-1/4. [088101].

https://doi.org/10.1103/PhysRevLett.100.088101

DOI:

10.1103/PhysRevLett.100.088101

Document status and date:

Published: 01/01/2008

\section{Document Version:}

Publisher's PDF, also known as Version of Record (includes final page, issue and volume numbers)

\section{Please check the document version of this publication:}

- A submitted manuscript is the version of the article upon submission and before peer-review. There can be important differences between the submitted version and the official published version of record. People interested in the research are advised to contact the author for the final version of the publication, or visit the $\mathrm{DOI}$ to the publisher's website.

- The final author version and the galley proof are versions of the publication after peer review.

- The final published version features the final layout of the paper including the volume, issue and page numbers.

Link to publication

\section{General rights}

Copyright and moral rights for the publications made accessible in the public portal are retained by the authors and/or other copyright owners and it is a condition of accessing publications that users recognise and abide by the legal requirements associated with these rights.

- Users may download and print one copy of any publication from the public portal for the purpose of private study or research.

- You may not further distribute the material or use it for any profit-making activity or commercial gain

- You may freely distribute the URL identifying the publication in the public portal.

If the publication is distributed under the terms of Article 25fa of the Dutch Copyright Act, indicated by the "Taverne" license above, please follow below link for the End User Agreement:

www.tue.nl/taverne

Take down policy

If you believe that this document breaches copyright please contact us at:

openaccess@tue.nl

providing details and we will investigate your claim. 


\title{
Accurate Determination of Elastic Parameters for Multicomponent Membranes
}

\author{
Stefan Semrau, ${ }^{1}$ Timon Idema, ${ }^{2}$ Laurent Holtzer, ${ }^{1}$ Thomas Schmidt, ${ }^{1}$ and Cornelis Storm ${ }^{2}$ \\ ${ }^{1}$ Physics of Life Processes, Leiden Institute of Physics, Leiden University, P.O. Box 9506, 2300 RA Leiden, The Netherlands \\ ${ }^{2}$ Instituut-Lorentz for Theoretical Physics, Leiden Institute of Physics, Leiden University, \\ P.O. Box 9506, 2300 RA Leiden, The Netherlands
}

(Received 28 February 2007; published 26 February 2008)

\begin{abstract}
Heterogeneities in the cell membrane due to coexisting lipid phases have been conjectured to play a major functional role in cell signaling and membrane trafficking. Thereby the material properties of multiphase systems, such as the line tension and the bending moduli, are crucially involved in the kinetics and the asymptotic behavior of phase separation. In this Letter we present a combined analytical and experimental approach to determine the properties of phase-separated vesicle systems. First we develop an analytical model for the vesicle shape of weakly budded biphasic vesicles. Subsequently experimental data on vesicle shape and membrane fluctuations are taken and compared to the model. The parameters obtained set limits for the size and stability of nanodomains in the plasma membrane of living cells.
\end{abstract}

DOI: 10.1103/PhysRevLett.100.088101

The recent interest in coexisting phases in lipid bilayers originates in the supposed existence of lipid heterogeneities in the plasma membrane of cells. A significant role in cell signaling and traffic is attributed to small lipid domains called "rafts" [1,2]. While their existence in living cells remains the subject of lively debate, micrometer-sized domains are readily reconstituted in giant unilamellar vesicles (GUVs) made from binary or ternary lipid mixtures [3]. Extensive studies of these and similar model systems have brought to light a rich variety of phases, phase transitions and coexistence regimes [4]. In contrast to these model systems, no large (micrometer-sized) membrane domains have been observed in vivo. If indeed phase separation occurs in vivo, additional processes which can arrest it prematurely must be considered. It has been suggested that nanodomains might be stabilized by entropy [5] or that, alternatively, active cellular processes are necessary to control the domain size [6]. A third explanation is that curvature-mediated interactions conspire to create an effective repulsion between domains, impeding and ultimately halting their fusion as the phase separation progresses. Each of these three processes depends critically on membrane parameters such as line tension [7], curvature moduli, and even the elusive Gaussian rigidities [8]. Although some studies report values [9] or upper bounds $[10,11]$ for these membrane parameters, a systematic method to determine them from experiments that does not require extensive numerical simulation and fitting is lacking. We present here a straightforward fully analytical method that allows for a precise, simultaneous determination of the line tension, the bending rigidity, and the difference in Gaussian moduli from biphasic GUVs. Both the liquid ordered $L_{o}$ and the liquid disordered $L_{d}$ phase are quantitatively characterized with high accuracy. Our method relies on an analytical expression for the shape of a moderately budded vesicle. A one-parameter fit to experimental shapes permits unambiguous determination of the line tension and the difference in Gaussian moduli. Our
PACS numbers: 87.16.D-, 02.40.Hw, 87.15.Ya, 87.17.Aa

results provide important clues as to the origin and magnitude of long-ranged membrane-mediated interactions, which have been proposed recently as an explanation for the trapped coarsening $[12,13]$ and the very regular domain structure of a metastable state [14] found in experiments. Furthermore, our results show that nanometer-sized phaseseparated domains will be stable in life cells.

Model. - The free energy associated with the bending of a thin membrane is described by the Canham-Helfrich free energy [15]. We ignore any spontaneous curvature of the membrane because the experimental system has ample time to relax any asymmetries between the leaflets. For a two-component vesicle with line tension $\tau$ between the components, the free energy then reads

$$
\mathcal{E}=\sum_{i=1,2} \int_{S_{i}}\left(2 \kappa_{i} H^{2}+\kappa_{G}^{(i)} K+\sigma_{i}\right) d A-p V+\tau \oint_{\partial S} d \ell,
$$

where the $\kappa_{i}$ and $\kappa_{G}^{(i)}$ are the bending and Gaussian moduli of the two phases, respectively, the $\sigma_{i}$ are their surface tensions, and $p$ is the internal pressure. In equilibrated shapes such as our experimental vesicles, the force of the internal Laplace pressure is compensated by the surface tensions; consequently, both contributions drop out of the shape equations [16]. For each phase, we integrate the mean $(H)$ and Gaussian $(K)$ curvature over the membrane patch $S_{i}$ occupied by that phase; the line tension contributes at the boundary $\partial S$ of the two phases. Using the Gauss-Bonnet theorem, we find that the Gaussian curvature term yields a constant bulk contribution plus a boundary term [17].

The axisymmetric shapes of interest (Fig. 1) are fully described by the contact angle $\psi$ as a function of the arc length $s$ along the surface contour. The coordinates $(r(s), z(s))$ are fixed by the geometrical conditions $\dot{r}=$ $\cos (\psi(s))$ and $\dot{z}=-\sin (\psi(s))$, where dots denote derivatives with respect to the arclength. Variational calculus 


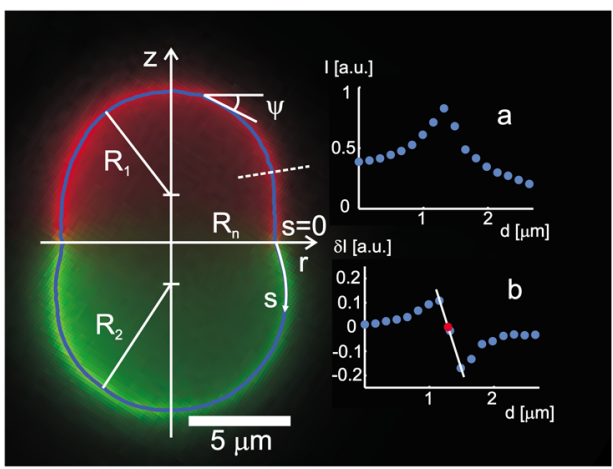

FIG. 1 (color online). Fluorescence raw data [red (or dark gray): $L_{o}$ domain; green (or light gray): $L_{d}$ domain] with superimposed contour [light blue (or gray)]. Insets: principle of contour fitting; (a) intensity profile normal to the vesicle contour (taken along the dashed line in the main image); (b) first derivative of the profile with linear fit around the vesicle edge (white line). The red (or dark gray) point marks the vesicle edge.

gives the basic shape equation [16]:

$$
\ddot{\psi} \cos \psi=-\frac{1}{2} \dot{\psi}^{2} \sin \psi-\frac{\cos ^{2} \psi}{r} \dot{\psi}+\frac{\cos ^{2} \psi+1}{2 r^{2}} \sin \psi .
$$

This equation holds for each of the phases separately. The radial coordinate $r(s)$ and tangent angle $\psi(s)$ must of course be continuous at the domain boundary. Additionally, the variational derivation of Eq. (2) gives two more boundary conditions [18]:

$$
\begin{aligned}
& \lim _{\varepsilon \downarrow 0}\left(\kappa_{2} \dot{\psi}(\varepsilon)-\kappa_{1} \dot{\psi}(-\varepsilon)\right)=\left(\Delta \kappa+\Delta \kappa_{G}\right) \frac{\sin \psi_{0}}{R_{n}}, \\
& \lim _{\varepsilon \downarrow 0}\left(\kappa_{2} \ddot{\psi}(\varepsilon)-\kappa_{1} \ddot{\psi}(-\varepsilon)\right)=-\left(2 \Delta \kappa+\Delta \kappa_{G}\right) \frac{\cos \psi_{0} \sin \psi_{0}}{R_{n}^{2}} \\
&+ \frac{\sin \psi_{0}}{R_{n}} \tau,
\end{aligned}
$$

with $R_{n}$ and $\psi_{0}$ the vesicle radius and tangent angle at the domain boundary, $\Delta \kappa=\kappa_{1}-\kappa_{2}, \Delta \kappa_{G}=\kappa_{G}^{(1)}-\kappa_{G}^{(2)}$, and the domain boundary located at $s=0$.

The sphere is a solution of Eq. (2); we can therefore use it as an ansatz for the vesicle shape far from the domain boundary. We split the vesicle into three parts: a neck domain around the domain boundary, where the boundary terms dominate the shape, and two bulk domains, where the solution asymptotically approaches the sphere. Perturbation analysis, performed by expanding Eq. (2) around the spherical shape, then gives for the bulk domains:

$$
\psi_{\text {bulk }}^{(i)}(s)=\frac{s+s_{0}^{(i)}}{R_{i}}+c_{i} R_{i} \log \left(\frac{s}{s_{0}^{(i)}}\right) .
$$

Here $R_{i}$ is the radius of curvature of the underlying sphere and $s_{0}^{(i)}$ the distance (set by the area constraint on the vesicle) from the point $r=0$ to the domain boundary. As was shown by Lipowsky [7], the invagination length, defined as the ratio $\xi_{i}=\kappa_{i} / \tau$ of the bending modulus and the line tension, determines the size of the neck region. Our three-domain approach applies when this invagination length is small compared to the size of the vesicle. At $s=$ $\xi_{i}$ the line tension, rather than the bending modulus, becomes the dominant term in the energy. Self-consistency of the solution requires that the deviation from the sphere solution at that point be small, i.e., given by the dimensionless quantity $\xi_{i} / R_{i}$. This fixes the integration constant $c_{i}$.

Near the domain boundary, $\psi$ must have a local extremum in each of the phases and we can expand it as

$$
\psi_{\text {neck }}^{(i)}(s)=\psi_{0}^{(i)}+\dot{\psi}_{0}^{(i)} s+\frac{1}{2} \ddot{\psi}_{0}^{(i)} s^{2} .
$$

The neck solutions must match at the domain boundary and also satisfy conditions (3) and (4). Moreover, they need to match the bulk solutions to ensure continuity of $\psi$ and its derivative $\dot{\psi}$. In total this yields seven equations for the eight unknowns $\left\{\psi_{0}^{(i)}, \dot{\psi}_{0}^{(i)}, \ddot{\psi}_{0}^{(i)}, s_{i}\right\}$. The necessary eighth equation is provided by the condition of continuity of $r(s)$ at the domain boundary.

Combined, the neck and bulk components of $\psi$ give a vesicle solution for specified values of the material parameters $\left\{\kappa_{i}, \Delta \kappa_{G}, \tau\right\}$. This solution compares extremely well to numerically determined shapes (obtained using the Surface Evolver package [19], Fig. 3). Moreover, for the symmetric case of domains with identical values of $\kappa$, we can compare to earlier modeling in Ref. [8]. The vesicle can then be described by a single dimensionless parameter $\lambda=R_{0} / \xi$, where $4 \pi R_{0}^{2}$ equals the vesicle area. The "budding transition" (where the broad neck destabilizes in favor of a small neck) is numerically found in Ref. [8] to occur at $\lambda=4.5$ for equally sized domains; our model gives a value of $\lambda=4.63$.

Experiment. - Giant unilamellar vesicles (GUVs) were produced by electroformation from a mixture of $30 \%$ DOPC, $50 \%$ brain sphingomyelin, and $20 \%$ cholesterol at $55^{\circ} \mathrm{C}$. Subsequently lowering the temperature to $20^{\circ} \mathrm{C}$ resulted in the spontaneous formation of liquid ordered $L_{o}$ and liquid disordered $L_{d}$ domains on the vesicles. The $L_{d}$ phase was stained by a small amount of rhodamine-DOPE $(0.2 \%)$. In order to stain the $L_{o}$ phase a small amount $(0.2 \%)$ of the ganglioside GM1 was added, and subsequently choleratoxin labeled with Alexa 647 was bound to the GM1 [20]. For imaging we chose a wide-field epifluorescence setup [2] because short illumination times (1-5 ms) prevent shape fluctuations with short correlation times from being washed out. The raw data of a typical vesicle are shown in Fig. 1. The lateral resolution of the equatorial optical sections was limited by diffraction and pixelation effects. In the normal direction, however, a high (subpixel) accuracy was obtained. The upper inset in Fig. 1 shows a typical intensity profile along a line perpendicular to the contour. We determine numerically the profile's first derivative (lower inset in Fig. 1) and fit the central part around the maximum intensity with a straight line. The 
intercept with the $x$ axis gives the position of the vesicle edge. The positional accuracy achieved is typically $20 \mathrm{~nm}$. The contours obtained were subsequently smoothed by a polynomial and all contours from the same vesicle (typically around 1000) were averaged to give the final result for the mean contour.

Spectra of the shape fluctuations were obtained from those parts of the contours that were nearly circular, i.e., far away from the neck domain. Fluctuations were determined for each single contour as the difference between the local radius $r$ and the ensemble averaged radius $R$ of a circle fitted to patches around the vesicles' poles: $u(s)=r(s)-$ $R$ where $s$ is the arclength along the circle; see Fig. 2. The experimental fluctuation spectrum was obtained by Fourier transform as $u_{k}=\frac{1}{a} \int_{-a / 2}^{a / 2} d s r(s) e^{-i k s}$, where $a$ is the arclength of the contour patch, and $k=n \frac{2 \pi}{a}$ with $n$ a nonzero integer. Taking into account the finite patch size [21] and following the spectral analysis of a closed vesicle shell developed by Pécréaux et al. [22] leads to a power spectrum for the vesicle fluctuation

$$
\overline{\left\langle\left|u_{k}\right|^{2}\right\rangle}=\sum_{q}\left(\frac{\sin \left((k-q) \frac{a}{2}\right)}{(k-q) \frac{a}{2}}\right)^{2} \overline{\left\langle\left|u_{q}\right|^{2}\right\rangle} \text { sph }
$$

Here $q=\frac{2 \pi}{L} m$ with $m$ a nonzero integer, $L=2 \pi R$, and $\overline{\left\langle\left|u_{q}\right|^{2}\right\rangle_{\text {sph }}}$ the spectrum of the entire vesicle derived in [22], where the overline indicates temporal averaging during the illumination time. Equation (7) was derived from the Canham-Helfrich free energy for a flat membrane with periodicity $L$. However, as shown in [22], the spectrum of a sphere with radius $R$ differs from that of the flat membrane only for the lowest wave numbers $k$. Therefore we can use Eq. (7) to fit our fluctuation spectra if we omit the two lowest modes. Examples of such fits are shown in Fig. 2.

Results. -Fits of the fluctuation spectra using Eq. (7) give the values of the bending moduli and surface tensions of the two phases. Using these values, we fit the experimentally obtained vesicle shapes with the model described

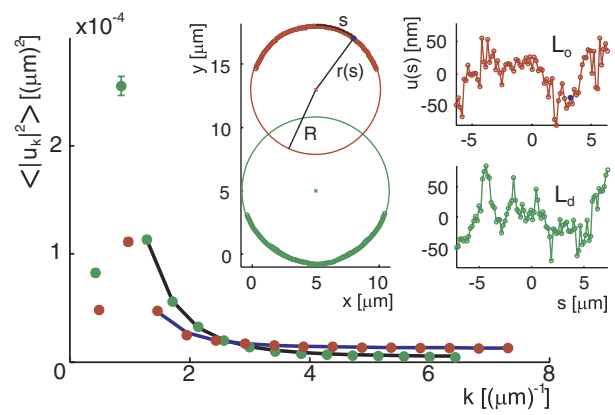

FIG. 2 (color online). Fluctuation spectra of the ordered [red (or dark gray) circles] and disordered [green (or light gray) circles] domains. The corresponding best fits of Eq. (7) are shown in blue (or gray) and black, respectively. Inset: Typical real-space fluctuations along the vesicle perimeter. above. This leaves us with two parameters: the line tension $\tau$ between the two phases and the difference $\Delta \kappa_{G}$ between their Gaussian moduli. Since the experimental data show that $\psi$ at the domain boundary follows a straight, continuous line we further assume that the derivative $\dot{\psi}$ is continuous at the domain boundary (as suggested before $[8,18]$ ). Imposing this additional condition fixes the value of $\Delta \kappa_{G}$ for given $\tau$, leaving us with a single free parameter to fully describe the system [23]. By fitting the experimental data, we directly extract the line tension. An example fit is shown in Fig. 3. Values found for the bending moduli are $8 \pm 1 \times 10^{-19} \mathrm{~J}$ for the $L_{o}$ domain and $1.9 \pm 0.5 \times$ $10^{-19} \mathrm{~J}$ for the $L_{d}$ domain. For the line tension we found a value of $1.2 \pm 0.3 \mathrm{pN}$, which is in the same range as that estimated by Baumgart et al. [14]. Finally, the difference in Gaussian moduli is about $3 \pm 1 \times 10^{-19} \mathrm{~J}$, in accordance with the earlier established upper bound $\left(\kappa_{G} \leq-0.83 \kappa\right)$ reported by Siegel and Kozlov [10]. An overview of the results is given in Table I.

Discussion. - Ultimately, one worries about the membrane's elastic parameters because their precise magnitude has important consequences for the morphology and dynamics of cells. The literature is replete with theoretical speculations which depend strongly on, among others, the line tension. While the values we report apply to reconstituted vesicles, we can nonetheless use them in some of these models to explore possible implications for cellular membranes. The majority of the investigated vesicles finally evolved into the fully phase separated state. This finding is in agreement with previous work by Frolov et al. [5], which predicts, for line tensions larger than $0.4 \mathrm{pN}$, complete phase separation for systems in equilibrium. It should be noted that the line tension found is also smaller than the critical line tension leading to budding: recent results by Liu et al. [24] show that for endocytosis by means of membrane budding both high line tensions $(>10 \mathrm{pN})$ and large domains are necessary. Therefore nanodomains will be stable and will not bud. In cells, however, additional mechanisms must be considered. To explain the absence of large domains in vivo, Turner et al.

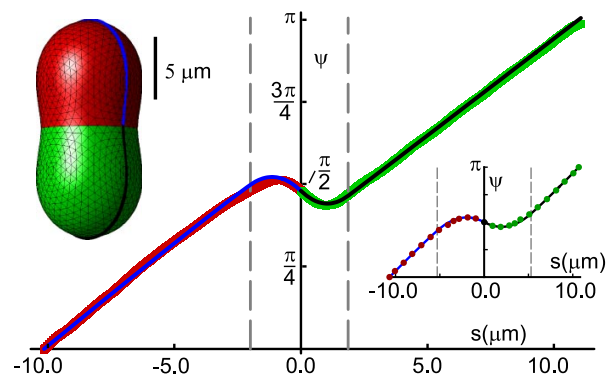

FIG. 3 (color online). Example for an experimentally obtained $\psi(s)$ plot [red (or dark gray): $L_{o}$ phase; green (or light gray): $L_{d}$ phase] together with the best fit of the model [blue (or gray): $L_{o}$ phase; black: $L_{d}$ phase]. The dashed lines mark the transition points between the neck and bulk domains. Insets: Fit to a numerically obtained shape (using Surface Evolver). 
TABLE I. Values of the material parameters for five different vesicles. The surface tensions and bending moduli of the $L_{d}$ and $L_{o}$ phase are determined from the fluctuation spectrum; the line tension and difference in Gaussian moduli are subsequently determined using our analytical model.

\begin{tabular}{ccccccc}
\hline \hline & $\begin{array}{c}\sigma_{d} \\
\left(10^{-7} \frac{N}{m}\right)\end{array}$ & $\begin{array}{c}\kappa_{d} \\
\left(10^{-19} \mathrm{~J}\right)\end{array}$ & $\begin{array}{c}\sigma_{o} \\
\left(10^{-7} \frac{N}{m}\right)\end{array}$ & $\begin{array}{c}\kappa_{o} \\
\left(10^{-19} \mathrm{~J}\right)\end{array}$ & $\begin{array}{c}\tau \\
(\mathrm{pN})\end{array}$ & $\begin{array}{c}\Delta \kappa_{G} \\
\left(10^{-19} \mathrm{~J}\right)\end{array}$ \\
\hline 1 & $2.8 \pm 0.2$ & $2.2 \pm 0.1$ & $0.3 \pm 0.3$ & $8.0 \pm 1.3$ & $1.5 \pm 0.3$ & $2.5 \pm 2$ \\
2 & $5.8 \pm 0.5$ & $1.8 \pm 0.2$ & $2.1 \pm 0.4$ & $8.2 \pm 1.5$ & $1.2 \pm 0.4$ & $2.0 \pm 2$ \\
3 & $3.5 \pm 0.3$ & $2.0 \pm 0.1$ & $2.0 \pm 0.5$ & $8.2 \pm 1.4$ & $1.2 \pm 0.3$ & $2.5 \pm 2$ \\
4 & $2.8 \pm 0.2$ & $1.9 \pm 0.1$ & $2.5 \pm 0.5$ & $8.3 \pm 1.2$ & $1.2 \pm 0.4$ & $4.0 \pm 2$ \\
5 & $2.3 \pm 0.1$ & $1.6 \pm 0.1$ & $0.6 \pm 0.3$ & $8.0 \pm 1.6$ & $1.1 \pm 0.5$ & $4.0 \pm 3$ \\
\hline \hline
\end{tabular}

[6] make use of a continuous membrane recycling mechanism. For the membrane parameters we have determined such a mechanism predicts asymptotic domains of $\sim 10 \mathrm{~nm}$ in diameter. Our results, in combination with active membrane recycling therefore support a minimal physical mechanism as a stabilizer for nanodomains in cells. A separate effect, purely based on the elastic properties of membranes may further stabilize smaller domains in vivo. Recently, Yanagisawa et al. explored the consequences of a repulsive interaction between nearby buds [12] and reported that such interactions can arrest the phase separation kinetics. The elastic perturbations induced by domains in the membrane, as described in this Letter, are obvious candidates for producing additional interactions between buds at any distance, further assisting in the creation of such a kinetic arrest. As Müller et al. have shown for a flat membrane, two distortions on the same side of an infinite flat membrane repel on all length scales [25]. The experimental observation of multiple domains ordered in (quasi-)crystalline fashion in model membranes [14] strongly suggests a similar repulsive interaction in spherical vesicle systems. This is indeed evidenced by preliminary numerical exploration of this system using Surface Evolver [19]. Membrane-mediated interactions act over length scales much larger than van der Waals or electrostatic interactions and could provide an alternative or additional physical mechanism for processes such as protein clustering and domain formation [13,26]. Our results and methods allow us not only to determine the parameters relevant to processes like these, but also give a practical analytical handle on the shapes involved. This, in turn, will help decide between competing proposals for mechanisms involving membrane bending: protein interactions, endocytosis, and the formation and stabilization of functional membrane domains.

This work was supported by funds from the Netherlands Organization for Scientific Research (NWO-FOM) within the program on Material Properties of Biological Assemblies (No. FOM-L1707M and No. FOM-L2601M).
[1] K. Simons and E. Ikonen, Nature (London) 387, 569 (1997); R. G.W. Anderson and K. Jacobson, Science 296, 1821 (2002); M. Edidin, Annu. Rev. Biophys. Biomol. Struct. 32, 257 (2003); F. R. Maxfield and I. Tabas, Nature (London) 438, 612 (2005); J.F. Hancock, Nat. Rev. Mol. Cell Biol. 7, 456 (2006).

[2] P. H. M. Lommerse, B.E. Snaar-Jagalska, H. P. Spaink, and T. Schmidt, J. Cell Sci. 118, 1799 (2005).

[3] C. Dietrich et al., Biophys. J. 80, 1417 (2001).

[4] S. L. Veatch and S. L. Keller, Phys. Rev. Lett. 94, 148101 (2005).

[5] V. A. J. Frolov, Y.A. Chizmadzhev, F. S. Cohen, and J. Zimmerberg, Biophys. J. 91, 189 (2006).

[6] M. S. Turner, P. Sens, and N. D. Socci, Phys. Rev. Lett. 95, 168301 (2005).

[7] R. Lipowsky, J. Phys. II (France) 2, 1825 (1992).

[8] F. Jülicher and R. Lipowsky, Phys. Rev. Lett. 70, 2964 (1993).

[9] T. Baumgart, S. Das, W. W. Webb, and J.T. Jenkins, Biophys. J. 89, 1067 (2005).

[10] D. P. Siegel and M. M. Kozlov, Biophys. J. 87, 366 (2004).

[11] J.-M. Allain, C. Storm, A. Roux, M. Ben Amar, and J.-F. Joanny, Phys. Rev. Lett. 93, 158104 (2004).

[12] M. Yanagisawa, M. Imai, T. Masui, S. Komura, and T. Otha, Biophys. J. 92, 115 (2007).

[13] B. J. Reynwar, G. Illya, V. A. Harmandaris, M. M. Müller, K. Kremer, and M. Deserno, Nature (London) 447, 461 (2007).

[14] T. Baumgart, S.T. Hess, and W.W. Webb, Nature (London) 425, 821 (2003).

[15] P. B. Canham, J. Theor. Biol. 26, 61 (1970); W. Helfrich, Z. Naturforsch. C 28, 693 (1973).

[16] J.-G. Hu and Z.-C. Ou-Yang, Phys. Rev. E 47, 461 (1993); W.-M. Zheng and J. Liu, Phys. Rev. E 48, 2856 (1993); F. Jülicher and U. Seifert, Phys. Rev. E 49, 4728 (1994).

[17] M. do Carmo, Differential Geometry of Curves and Surfaces (Prentice-Hall, Englewood Cliffs, NJ, 1976).

[18] F. Jülicher and R. Lipowsky, Phys. Rev. E 53, 2670 (1996).

[19] K. Brakke, Exp. Math. 1, 141 (1992).

[20] The DOPC (1,2-di-oleoyl-sn-glycero-3-phosphocholine), sphingomyelin, cholesterol, rhodamine-DOPE [1,2dioleoyl-sn-glycero-3-phosphoethanolamine-N-(lissamine rhodamine B sulfonyl)], and GM1 were obtained from Avanti Polar Lipids; the Alexa labeled choleratoxin from molecular probes.

[21] M. Mutz and W. Helfrich, J. Phys. (France) 51, 991 (1990).

[22] J. Pécréaux, H.-G. Döbereiner, J. Prost, J.-F. Joanny, and P. Bassereau, Eur. Phys. J. E 13, 277 (2004).

[23] A two-parameter fit without the continuity condition on $\dot{\psi}$ at the domain boundary gives the same results within the experimental accuracy.

[24] J. Liu, M. Kaksonen, D. G. Drubin, and G. Oster, Proc. Natl. Acad. Sci. U.S.A. 103, 10277 (2006).

[25] M. M. Müller, M. Deserno, and J. Guven, Europhys. Lett. 69, 482 (2005).

[26] H.T. McMahon and J.L. Gallop, Nature (London) 438, 590 (2005); J. Zimmerberg and M. M. Kozlov, Nat. Rev. Mol. Cell Biol. 7, 9 (2006). 